\title{
Analyzing the Theoretical Performance of Information Sharing
}

\author{
Paul Scerri, Prasanna Velagapudi and Katia Sycara
}

\begin{abstract}
Individuals in large, heterogeneous teams will commonly produce sensor data that is likely useful to some other members of the team, but it is not precisely known to whom the information is useful. Some recent work has shown that randomly propagating the information performed surprisingly well, compared to infeasible optimal approaches. This chapter extends that work by looking at how the relative performance of random information passing algorithms scales with the size of the team. Additionally, the chapter looks at how random information passing performs when sensor data is noisy, so that individuals need multiple pieces of data to reach a conclusion, and the underlying situation is dynamic, so individuals need new information over time. Results show that random information passing is broadly effective, although relative performance is lower in some situations.
\end{abstract}

\section{Introduction}

Exciting applications are emerging that involve large, heterogeneous teams acting in complex environments. Examples include search and rescue [4], disaster response [13], and military applications [3]. In such domains, team members will often collect local information that is necessary or useful to other members of the team. For example, in urban search and rescue operations, an aerial robot might be able to locate a victim, but be unable to assess their condition or determine a route to them. A ground robot on the team could perform these tasks, but might be unable to locate the victim by itself. Efficiently getting such information from those collecting

Paul Scerri

Carnegie Mellon University, Pittsburgh, PA 15217, USA, e-mail: pscerri@cs.cmu.edu

Prasanna Velagapudi

Carnegie Mellon University, Pittsburgh, PA 15217, USA, e-mail: pkv@cs.cmu.edu

Katia Sycara

Carnegie Mellon University, Pittsburgh, PA 15217, USA, e-mail: katia@cs.cmu.edu 
it to those requiring it is one of the keys to effective team performance. However, teammates often have limited information about which, if any, team members require particular pieces of information. Thus, the team member collecting some piece of information needs to determine whether and where to send collected information with limited knowledge of who might need it and how important it is to them. At the same time, team members must also be careful about what they communicate as the volume of incoming information is typically dramatically higher than available communication bandwidth.

Recognizing the utility of such information and delivering it efficiently across a team has been the focus of much research, with proposed approaches ranging from flooding [2] to channel filters [1] and matchmakers [7]. Interestingly, random forwarding of information has been found to be a surprisingly effective information sharing approach in some domains [2]. In previous work, we investigated this phenomena in detail and showed that, in certain systems, random forwarding of information performs almost half as well as a globally optimal approach [15].

In small teams or static environments, a variety of approaches have been applied to the information sharing problem. One example, STEAM [14], requires team members to keep others informed of their current state, allowing members to intelligently reason about which teammates need which information. Approaches using matchmakers [7] allow team members to keep some central point informed of their state, while the control point is responsible for directing information as required. More recently, for applications such as sensor networks, algorithms drawing on intuitions of how human gossip [2] works have been shown to be effective, but often wasteful with bandwidth. Token-based algorithms have also been shown to be effective for large-scale team coordination [17] and belief sharing [15] in some domains.

An interesting feature of both gossip and token algorithms is that little knowledge of the team is known or assumed. Nearly random communication coupled with local reasoning is sufficient to produce surprisingly competitive results [16]. It is this surprising effectiveness of lightweight, decentralized, and largely random algorithms that is the focus of this paper. The intention in this work is to understand and quantify when and how these simple strategies will be effective.

In previous work, we established an upper-bound on average case performance of information sharing in large teams and showed that in certain circumstances random policies can achieve a significant portion of that performance [16]. By adding simple heuristics to avoid redundant communications, it was possible to improve the performance of a purely random policy significantly. This means that in domains where network and utility distributions are similar to these cases, random information sharing policies may present an efficient and robust information sharing solution.

This paper extends that previous work in two important ways. First, it looks at how the relative performance of the random policies vary with the size of the network. Two opposing forces influence the performance. On the one hand, bigger networks allow random strategies to revisit the same agent less often, improving their relative performance, but bigger networks give more options for an intelligent strategy to exploit, reducing the relative performance of random strategies. Second, this paper looks at cases where agents might need more than one piece of informa- 
tion, either because the information is noisy or because the environment is dynamic. In one case, there are multiple, overlapping, noisy sensor readings generated by the team over time. Here, even team members with very high interest in a particular piece of information will not need to know every reading, only a subset that allows the underlying features or state to be inferred correctly. In another case, the underlying environment changes over time and agents need to track features. Results show that random information propagation is relatively better in the noisy case, but relatively worse in the dynamic case.

\section{Information Sharing}

In this section, we formally describe the information sharing problem. Consider a team of agents, $A$, working to achieve some goal. Suppose there is a piece of information $\eta$ obtained by team member $a \in A$. If any other member $b \in A$ were to obtain that information, it would impact their ability to perform the team goal. Define the utility $\xi_{a}(\eta)$ as the quantification of this change in performance. In order for $b$ to get the information, it must be communicated across the network, $N$. This requires some members of the team to spend resources such as time and power on communicating the information. Thus, there is some communications cost $\kappa(a, b, \eta)$ associated with transmitting $\eta$ from $a$ to $b$.

The best team performance is achieved when information is shared with the set of team members that have a higher utility for the information than the cost of communicating it to them. If the communication cost is expressed in the same units as the utility, this is represented by the maximization:

$$
A^{*}=\arg \max _{A \subseteq T} \sum_{a \in A}\left(\xi_{a}(\eta)-\kappa(\cdot, a, \eta)\right)
$$

In order to address the specific problem of information sharing in a large, dynamic team, we make several key assumptions. First, communications are assumed to be peer-to-peer and of a constant cost per transmission. Rather than representing the communications cost of every pair of agents, $\kappa(a, b, \eta)$ can be compactly represented as some fixed cost $\kappa$ when agents are neighbors in the network, and infinite when they are not. This is reasonable for domains with peer-to-peer communications, as transmission between distant teammates in a network can be decomposed into a sequence of transmissions to their intermediate neighbors. The only solutions that are lost in this decomposition are solutions where teammates forward information along but do not make use of it, a rare case for a team.

Given this assumption, it is possible to condition the performance of an information sharing algorithm by the number of communications it has used. In many domains, the tradeoff between communication cost and utility is not well characterized or fixed. Avoiding it allows results to be generalized by removing the second term from Equation (1) and allowing us to compare performance across algorithms which make multiple communications per time step. 
Second, while the utility of some information will change over time, we assume that communication is sufficiently fast that utility is constant while a single piece of information is being shared across the network. For example, in a search and rescue domain, the utility of knowing where a fire is will change if the fire spreads or team members move relative to it. However, the speed of these changes is orders of magnitude slower than the millisecond scale transmission speeds of a modern wireless network connecting the team members.

Finally, in a large team, rather than modeling the utility explicitly for each member, we assume that it can be summarized in a utility distribution over all agents. This distribution represents the probability that team member $A$ has some utility $\xi$ for a piece of information $\eta$. For a given domain, the utility distribution can be computed empirically by conditioning on relevant variables and sampling utility as information is shared in the team. For analytic purposes, we can approximate this distribution by a number of canonical probability distributions such as normal, uniform, and exponential distributions.

\subsection{Token Algorithms}

Given these assumptions, we consider two extremes of token-based algorithm design in addressing this problem. In these algorithms, a token is created that contains some information $\eta$. This token is atomically passed from teammate to teammate. When a team member receives the token, it can make use of the information inside, then decide to either forward the token to a neighbor or delete it. Since tokens use exactly one communication per time step, token algorithms can control the number of communications by using tokens that are deleted after a fixed number of steps.

If we take advantage of all possible knowledge of agent utility and network properties, the optimal approach is to directly solve the maximization in Equation (1). This is done using an exhaustive search of all possible network paths of length $t$. We call this a $t$-step lookahead approach.

On the other hand, if we ignore all available knowledge of agent utility, we can propose a simple algorithm of randomly passing information from neighbor to neighbor. This equates to simply performing a random walk across the network. We therefore call this the random walk approach.

Given that no knowledge of utility is used in routing a random walk, its efficiency is primarily determined by its coverage of the network. We therefore introduce two intermediate algorithms that are equally naïve with regards to utility, but significantly more intelligent about coverage. A token will maximize coverage if it never revisits the same agent in a network. Thus, a straightforward improvement to the random walk approach is the addition of a history of nodes carried within the token. As the token moves around the network, visited agents are marked in this history, and when the token is being routed, this history is used to exclude visited agents from selection. If all neighbor nodes are visited, the algorithm selects a link at ran- 
dom. This approximates a self-avoiding walk over the network, so we term this the random self-avoiding walk approach.

This approach is reasonable when the size of the history is expected to be bounded to a reasonable size. However, in very large teams or systems where tokens visit many agents of the team, this is not a practical solution. In these cases, if there are a bounded number of tokens in existence at any given time, an alternative solution is to maintain a local history at each agent for each active token, consisting of its previously used incoming and outgoing network connections. Similarly to the random self-avoiding approach, agents that receive a token multiple times will attempt to send it to different neighbors each time, selecting randomly from the outgoing edges if all of them have been previously used. We designate this the random trail approach.

\section{Experimental Results}

A highly abstracted information sharing token simulator was created to empirically test the performance of token-based information sharing methods. The simulation consists of a network of agents that are assigned utilities for a given piece of information from a specified distribution. A token representing that information is initialized at a randomly chosen agent within the network. The agents propagate the token around the network according to some routing policy, and the accumulated utility is recorded at each step until the simulation executes some fixed number of steps. Results are averaged over 20 runs.

Five canonical network types were examined: small-worlds, scale-free, hierarchical, lattice, and random. Unless otherwise stated, each was generated to contain 1000 nodes with an average degree of 4 . The small-worlds network was generated by adding random links to a doubly-connected ring. The scale-free network was generated using a tunable variant of the Barabási-Albert algorithm [9]. The hierarchical network was formed by adding nodes evenly to a balanced tree. The lattice was a four-connected 2D grid with wraparound, and the random network was created by adding random links until the average degree was reached. In most cases, results for random networks were analogous to those of scale-free networks, thus some results for random networks were omitted for brevity.

Three canonical distributions were examined: uniform, normal, and exponential. The uniform distribution was over the interval $[0,1]$. The exponential distribution had a rate parameter of $\lambda=1.0$, but was scaled by a factor of 0.2 . In the case of the normal distribution, the mean and variance of the distribution were sometimes altered for various trials, but the nominal parameters were $\mu=0.5, \sigma=0.2$.

Four information sharing methods were considered: optimal, random walk, random trails, and self-avoiding walks. The optimal policy was approximated using a finite lookahead policy with global knowledge. Every $m$-steps, an exhaustive search of paths of length $m$ was executed to determine an optimal path. This path was executed fully, followed by another $m$-step planning phase. Ideally, this stage would 
consist of a single path search of the final path length, but computing this path is extremely expensive due to the non-Markovian nature of the utility function (as agents are visited, their utility drops to zero, so the joint distribution of utility is always dependent on complete network state). Instead, smaller values of $m$ were chosen empirically from the results of early experiments.

\subsection{Optimality of the Lookahead Policy}

In order to determine a sufficient approximation of optimality, an experiment was conducted in which the depth of the lookahead policy was varied over the four network types with a normal utility distribution $(\mu=0.5, \sigma=0.2)$, and the utilities of the resulting communication paths computed. The results of these tests can be seen in Figure 1, where the utility obtained by each token is plotted against the number of communications the token was allowed. As lookahead depth increases, the obtained utility converges asymptotically to the optimal. Interestingly, while lookahead depths of 1, 4 and 8 converge toward an asymptote, 2-step lookaheads, denoted by the circle symbols, appear to perform pathologically poorly. It is possible that this is due to a negative interaction between the width of the networks and the depth of the search pattern, where the lookahead policy may consistently make myopic routing decisions. From these results, a lookahead policy with a depth of 4 was selected as a baseline for future experiments, as a compromise between computational complexity and optimality of performance.

It is also possible to evaluate the optimality of token-based lookahead policies against the upper bound established in [16]. The dashed lines in Figure 1 correspond to these bounds. Two key characteristics are immediately evident. First, the lookahead policies often converge very closely to the upper bound on performance, suggesting that in the ideal case, token routing methods can perform very close to optimal. Second, while the bound is independent of network structure, clear differences are visible in the optimality of the lookahead policy over different network types. Most notably, in Figure 1d, the hierarchical network performs much worse than the upper bound, suggesting that information propagation via tokens in this type of structure is either highly inefficient or requires an extremely deep lookahead depth.

\subsection{Optimality of the Random Policies}

The four information sharing methods were tested in normal and exponential distributions. Figures 2 and 3 show the results of these experiments. It is evident that performance is heavily influenced by network type, with all policies performing significantly worse in hierarchical networks with both distributions and random policies performing proportionally much worse in the small worlds and hierarchical 


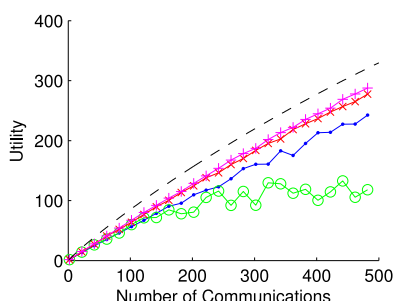

(a) Small worlds

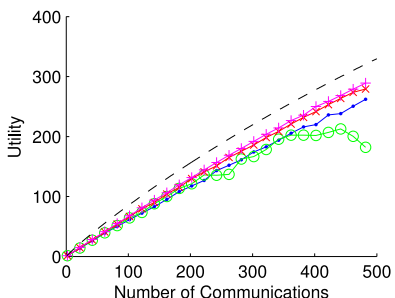

(c) Lattice

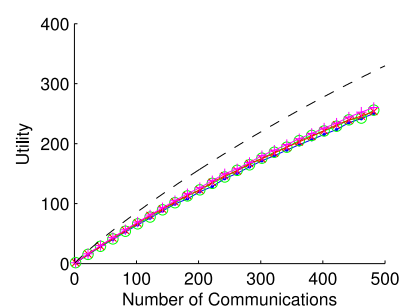

(b) Scale-free

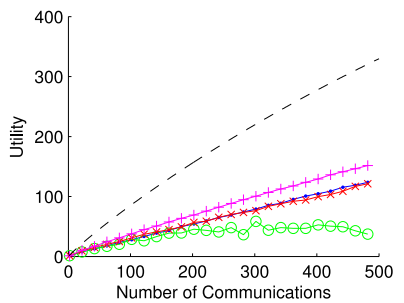

(d) Hierarchical

$$
\begin{array}{|r|r|}
\hline \text { - } 1 \text {-step } & 1-\text { 8-step } \\
-\quad \text { 2-step } & --- \text { Upper Bound } \\
\hline & \text { 4-step }
\end{array}
$$

Fig. 1: Optimality of n-step lookahead over four network types with a normal utility distribution $(\mu=0.5, \sigma=0.2)$. The utility obtained by each token is plotted against the number of communications used.

networks (Figures $2 \mathrm{~d}$ and $3 \mathrm{~d}$ ). Random policies perform best in the scale-free and lattice networks, with purely random walks attaining almost half the utility of the lookahead policy in the scale-free network with a normal utility distribution. The addition of self-avoiding heuristics appears to improve random policy performance significantly, primarily in the lattice and scale-free networks. The similar performance of the random trail and self-avoiding walk policies suggests that they are comparably effective at avoiding previously visited agents when covering the network. Further experiments focused on the random trail policy, as it typified the performance of the heuristic random policies.

As an example of the surprisingly efficient performance of random policies, consider Figure 2b. In it, we find that a utility of 175 is attained by a lookahead policy using an average of 300 communications. The same utility is obtained by a random self-avoiding policy in 425 communications. However, the random self-avoiding policy has no computational or structural overhead as it is completely unaware of utility. This suggests that under these conditions, if the cost of maintaining the necessary knowledge to perform an optimal strategy is on par with the cost of the extra 125 communications, a randomized policy is in fact a competitive strategy for information sharing. 


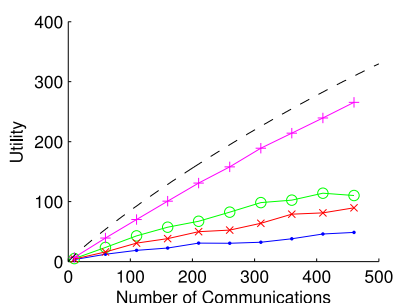

(a) Small worlds

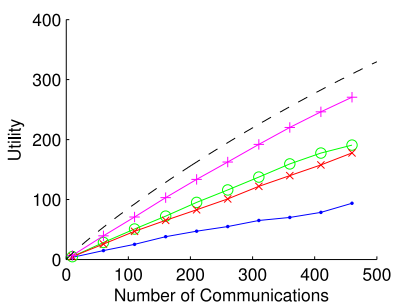

(c) Lattice

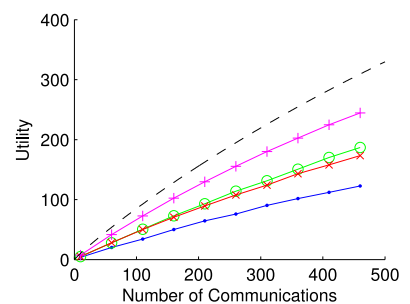

(b) Scale-free

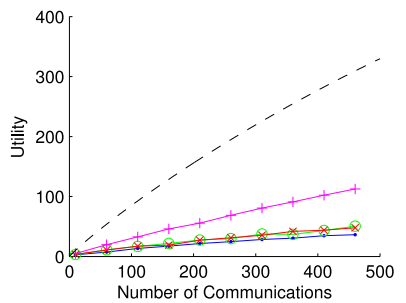

(d) Hierarchical

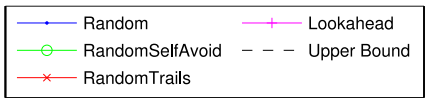

Fig. 2: Performance of random and lookahead policies over four network types with a normal utility distribution $(\mu=0.5, \sigma=0.2)$. The utility obtained by each token is plotted against the number of communications used.

\subsection{Effects of Noisy Estimation}

Exploring this tradeoff further, we examine the effects of noisy estimates of utility on performance of the lookahead policy. Gaussian noise was introduced into the utility estimates of unvisited team members used by the lookahead policy. The utility of visited members was fixed at zero and not affected by this noise. To simulate the compounded inaccuracy of estimating the utility of team members further away in the network, the standard deviation of the noise $(\gamma)$ was scaled exponentially by the network distance between teammates $\left(d_{a, b}\right)$ using the following equation.

$$
\gamma_{a, b}=(\gamma+1.0)^{d_{a, b}}-1.0
$$

As seen in Figure 4, as the amount of noise was increased, the performance of the lookahead policies degraded. This suggests that even when using an ideal routing policy, incorrect estimates of utility can disrupt intelligent routing policies. However, at $\gamma=1.0$, the noise was so large that estimates of utility were approximately random. The only usable information available in this condition was that the utility of visited teammates was fixed at zero. Without the ability to discern high- and low-utility team members, the remaining difference in performance between the 


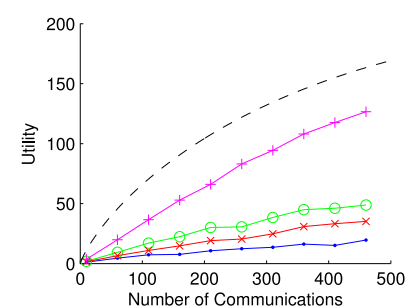

(a) Small worlds

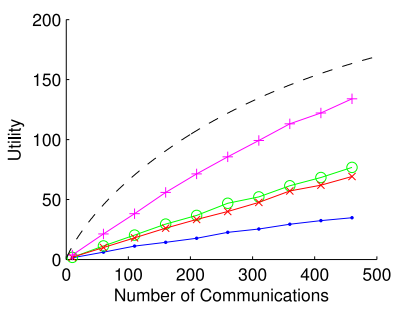

(c) Lattice

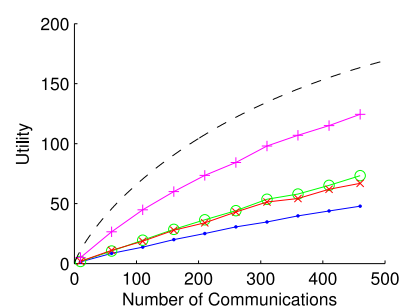

(b) Scale-free

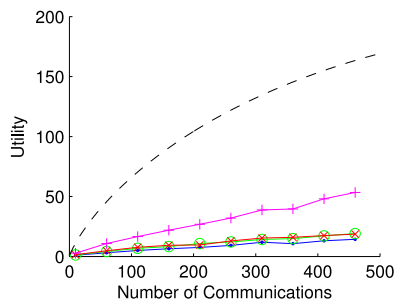

(d) Hierarchical

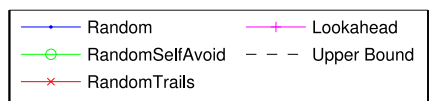

Fig. 3: Performance of random and lookahead policies over four network types with an exponential utility distribution $(\lambda=1.0$, scale factor of 0.2$)$. The utility obtained by each token is plotted against the number of communications used.

lookahead and random policy in the high noise condition cannot be attributed to the selection of higher utility paths. However, it may be the result of the lookahead policy's ability to avoid myopic routing decisions that might force future communications to pass through visited teammates.

\subsection{Properties Affecting Optimality}

Another observation was that the proportional gap between the lookahead policy and the random trail policy varied repeatably over networks and utility distributions across trials, suggesting that a combination of network structure and utility distribution properties affect the efficiency of the random trail policy. To explore this further, a wide array of networks and utility distributions were tested across a constant number of communications of $t=250$ to study how the optimality of the random trail policy was affected by various properties. A cross section of these results can be found in Figures 5 and 6 . It was found that certain characteristics clearly affected optimality, while most had negligible effects. 


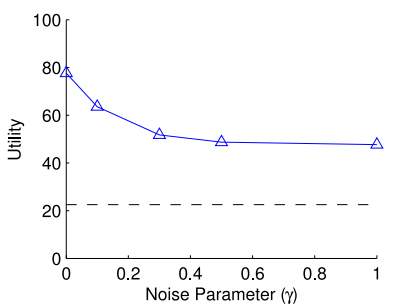

(a) Small worlds

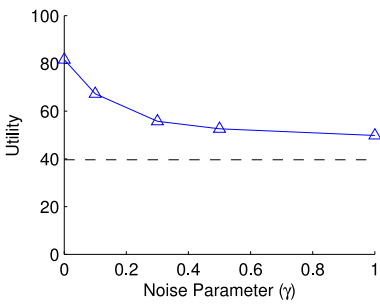

(c) Lattice

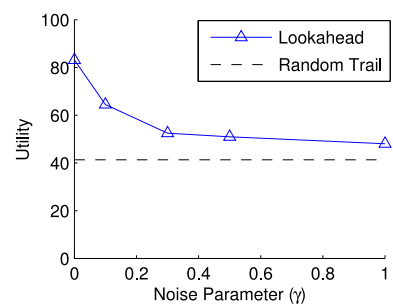

(b) Scale-free

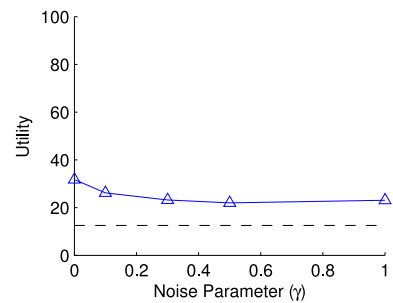

(d) Hierarchical

Fig. 4: Effects of noise on lookahead and random trail policy over four network types with a normal utility distribution $(\mu=0.5, \sigma=0.2$ ). The utility obtained by each token is plotted against a noise scaling parameter $\gamma$.

The type of network had a clear impact on optimality. Interestingly, small worlds and hierarchical networks were similar in performance and contrasted with scalefree networks. In this experiment, particularly interesting results were obtained for random networks, so these results are presented in lieu of the grid results.

The network density was another property that showed a clear effect on optimality. At low network densities $(\rho=2)$, the average case performance of the random trail algorithm matched or exceeded the optimal policy on the small worlds and random networks. The consistency of this result, and its specificity, suggest that certain combinations of network structure, utility distribution, and network density are pathological for the lookahead policy. At higher densities, the optimality seemed to converge to a constant value dependent on network type.

Aside from inconsistent behavior at low network densities, the variance of the utility distribution also affected optimality, with the random trail policy performing better as variance was decreased. This makes sense, as a perfect self-avoiding policy over a network of members with constant utility (no variance) will always take an optimal path. 


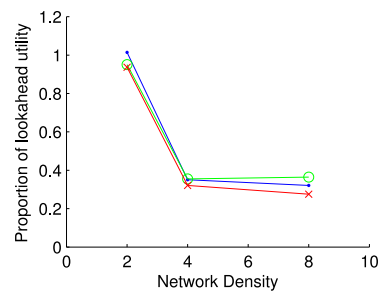

(a) Small worlds

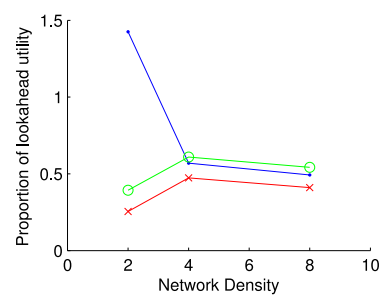

(c) Random

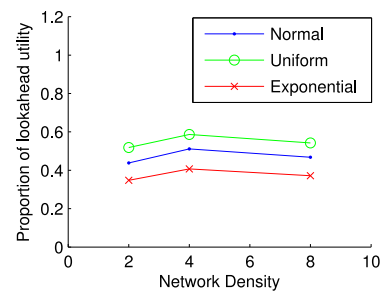

(b) Scale-free

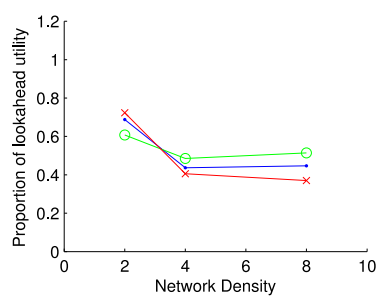

(d) Hierarchical

Fig. 5: Effects of network density on optimality of random trail policy over four network types with a normal $(\mu=0.5, \sigma=0.2)$, exponential $(\lambda=1.0$, scale factor of 0.2$)$, and uniform utility distribution. The proportion of lookahead utility refers to the utility of the random trail policy scaled by that of a 4-step lookahead policy.

\subsection{Scaling Network Size}

In the initial set of scaling experiments, distribution type, routing type and network type were varied as the size of the network was scaled up. The results are shown in Figures 7-9. Each of the figures use log scaling on both the $x$ and $y$ axes. In the first of the experiments, varying the distribution type, random trails routing was used on a scale free network (Figure 7). In all cases, overall value for a fixed token propagation length is shown. The slight improvement in value received with bigger networks was due to the larger network resulting in less situations where the token unavoidably retraced its own path. Notice that this effect lessens as the network gets bigger and will asymptotically reach some limit when it never retraces it path. In the second of the experiments, the routing type was varied on a scale free network, with an exponential value distribution, while the network size was scaled up. Notice that the lookahead policy, which knows the whole network improves its value much more with the increasing network size than any of the random policies. This is because the larger network offers more opportunities for the lookahead to exploit. Since the scale-free network has a small worlds property, the width of the network, i.e., the average distance between any two nodes in the network increases only slowly, thus offering many more opportunities for the lookahead to exploit, at relatively low cost. While the random policies benefit from retracing their steps less often, they do not proactively exploit the new opportunities. In the third scaling experiment, 


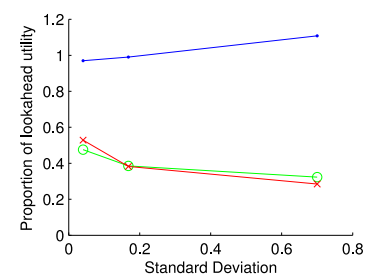

(a) Small worlds

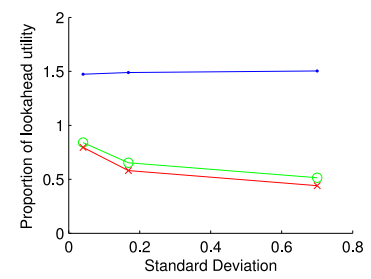

(c) Random

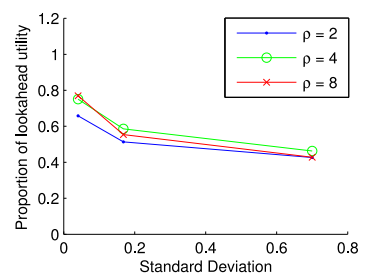

(b) Scale-free

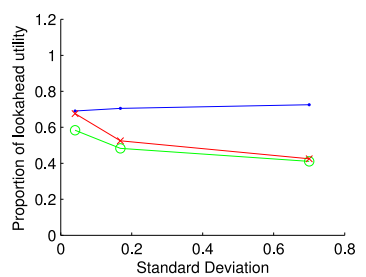

(d) Hierarchical

Fig. 6: Effects of variance on optimality of random trail policy over four network types with a normal utility distribution $(\mu=0.5, \sigma$ varied) and varying network densities. The proportion of lookahead utility refers to the utility of the random trail policy scaled by that of a 4-step lookahead policy.

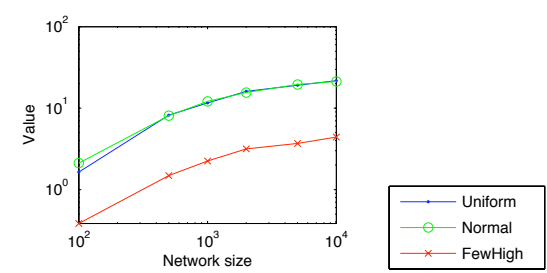

Fig. 7: Effects of varying value distribution as a scale free network is scaled up on relative optimality of random policies.

the network type is varied, with random trails routing and an exponential value distribution, while the network size is scaled up. The improvement in value for the bigger networks was much less pronounced for the hierarchy, because the structure of that network does not make it easy for the token to avoid revisiting agents, even in very big hierarchies. The other network types offer many more alternative paths, hence lead to a bigger value increase for the bigger network.

To confirm that it is the reduced need to revisit agents that allows the value increase with scale up for the random routing policies, we varied the network density of a scale free network and used random trails routing on the networks, while the overall network size was scaled up. The results are shown in Figure 10. Notice that for the larger networks, random trails performed relatively better on denser networks (average density 4 or 8 ) than on sparser networks (average density 2), confirming the hypothesis. There is no significant difference in the average density 4 and aver- 


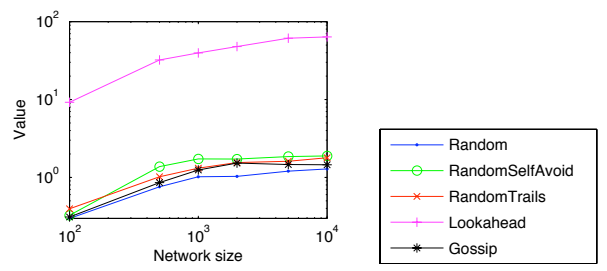

Fig. 8: Effects of varying routing type as a scale free network is scaled up on relative optimality of random policies.

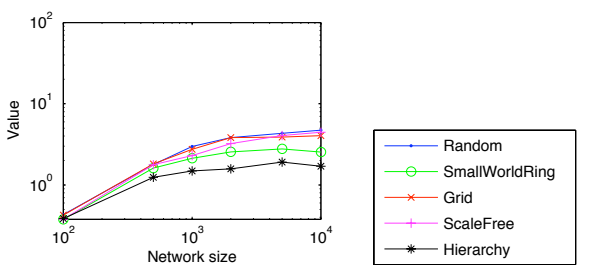

Fig. 9: Effects of varying network type as a scale free network is scaled up on relative optimality of random policies.

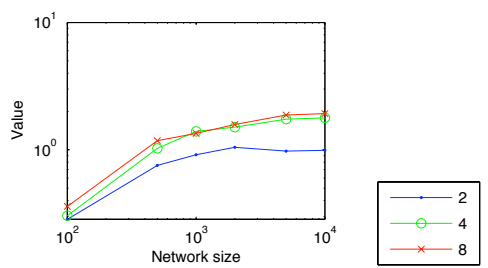

Fig. 10: Confirming the impact of revisits on random policies by varying network density of a scale free network with network size scale up.

age density 8 case, because the token already has sufficiently many options in the density 4 case to avoid most revisits.

The next set of experiments looked at the performance of the lookahead routing on small-worlds networks and hierarchies as the network density is increased (see Figure 11 - 14). The network density makes relatively little difference to overall effectiveness, since these social networks have relatively low width, even when the density is quite low. The line labeled " 0.20 .7 " uses a normal distribution of values with a mean value of 0.2 and a standard deviation of 0.7 , and similarly for the other lines. As expected, when the mean is higher, the lookahead is able to find better agents to deliver the information to and when the standard deviation is higher, the lookahead exploits the high value agents efficiently.

Finally, we looked at how random routing performs when an agent might need multiple pieces of information. Two possibilities were modeled. First, when propagated information was noisy sensor readings, with agents potentially needing multiple readings to reduce uncertainty sufficiently. Second, when information was prop- 


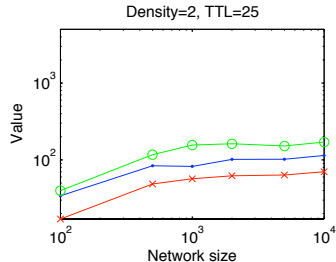

(a) Density $=2$

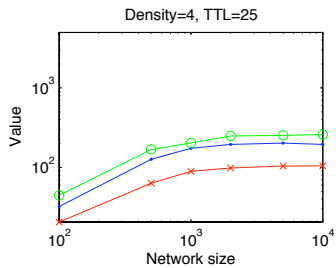

(b) Density $=4$

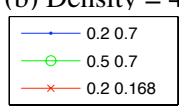

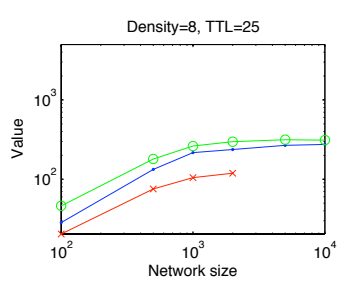

(c) Density $=8$

Fig. 11: The performance of the lookahead algorithm on small worlds networks of varied density, with $\mathrm{TTL}=25$.

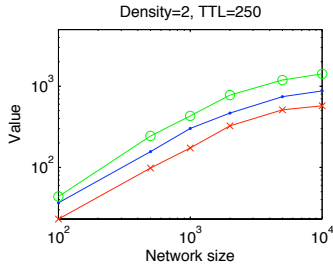

(a) Density $=2$

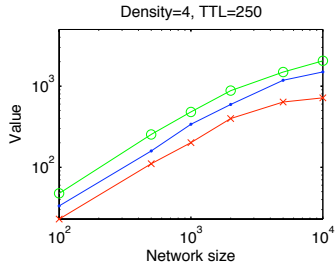

(b) Density $=4$

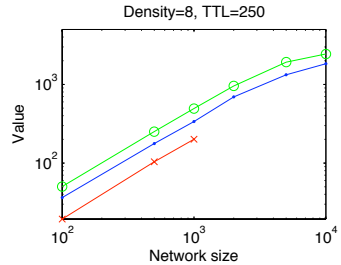

(c) Density $=8$

Fig. 12: The performance of the lookahead algorithm on small worlds networks of varied density, with $\mathrm{TTL}=250$.

agated about something that was changing over time, hence requiring new information occasionally. Measuring the average value received by the tokens was not an appropriate metric, in this case, because it incentivized behavior where there was maximal delay between delivering dynamic information to agents that needed it most, since that would be high value. Instead, the performance metric used was the sum of information needs over both agents and time. For this metric, lower is better, since lower implies agents needing information the most have received it earliest (and more often, in the dynamic case.) Figure 15 shows the results. In the dynamic case, the relative advantage of lookahead over random policies increases almost linearly, since the lookahead policy can repeatedly exploit its search to find agents with highest value for the information. However, in the noisy case, the difference gets smaller over time because there is less value for the lookahead to exploit after the highest value agents have received the information. Notice that in this case, the random trails and random self avoid policies do not attempt to avoid repeated visits across tokens, only for the single token. 


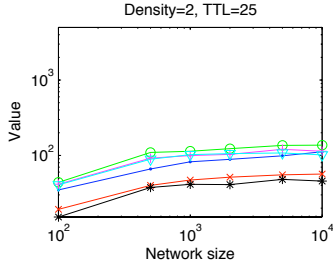

(a) Density $=2$

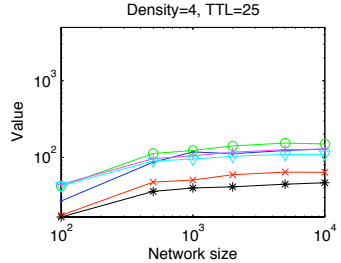

(b) Density $=4$

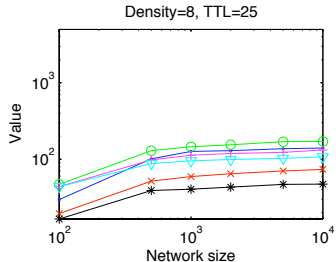

(c) Density $=8$

Fig. 13: The performance of the lookahead algorithm on hierarchies of varied density, with TTL = 25 .

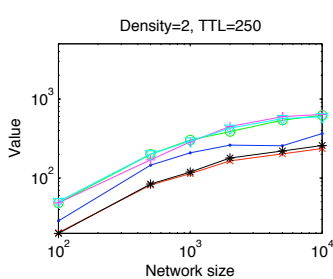

(a) Density $=2$

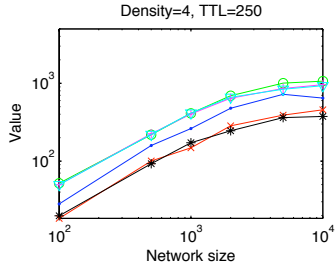

(b) Density $=4$

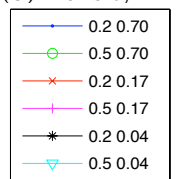

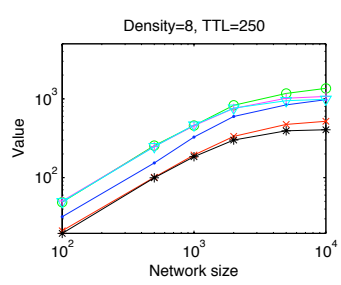

(c) Density $=8$

Fig. 14: The performance of the lookahead algorithm on hierarchies of varied density, with TTL $=$ 250 .

\section{Related Work}

The problem of communication in teams has been well-studied in a variety of fields. Approaches such as STEAM [14] and matchmakers [8] share knowledge about information requirements in order to reason about where to direct information. Gossip algorithms [2] and token passing algorithms [17, 15] use randomized local policies to share information and are thus particularly suited to large scale problems. To address the expense of synchronizing beliefs over teams, several techniques have been developed in conjunction with decentralized Bayesian filtering techniques, including channel managers [1] and query-based particle filters [11].

A number of approaches to communication for multi-agent coordination have evolved around the concept of multi-agent partially observable Markov decision 


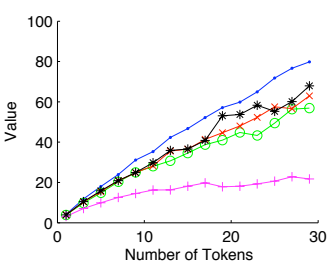

(a) Dynamics

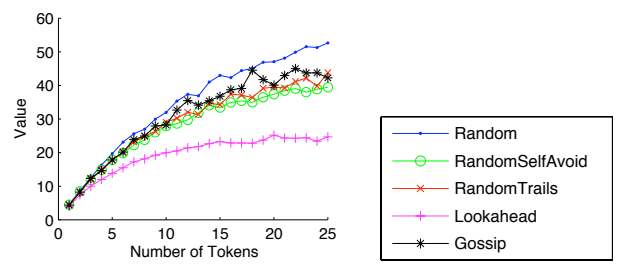

(b) Noise

Fig. 15: The relative performance of random strategies when multiple pieces of information are required per agent, either due to noise or dynamics on a scale free network.

processes (POMDPs). Some approaches augment the decentralized problem with actions for synchronization [10], while others model communications as an explicit choice and seek to maximize the tradeoff between communication cost and reward $[18,5]$ to achieve goals such as minimizing coordination errors [12].

\section{Conclusions and Future Work}

This chapter extends previous work that established upper bounds on token-based propagation. Specifically, it was shown the that relative performance of random token-passing policies scaled well with overall network size and were actually relatively better when noisy. Overall random policies were found to perform relatively poorly on small-worlds networks, while performing well on scale-free and lattice networks. In addition, hierarchical networks were shown to be ill-suited to even optimal token-based information sharing algorithms. The empirical conclusions support the idea that for some domains, random information sharing policies will be a very reasonable approach to take.

In future work, we will apply these results to a variety of physical domains, including urban search and rescue and mobile mesh networking. Using these analysis methods, it should be possible to determine which information sharing methods are best suited to these domains, including if and when random policies should be used. In addition, by modeling the utility distributions of these domains, it may be possible to gain insight into the fundamental properties of real-world information sharing problems, in turn improving the information sharing algorithms that must address them. Further graph-theoretic and probabilistic analysis should yield tighter bounds on performance, and additional experiments can determine the optimality of other common information sharing algorithms such as classic flooding [6], gossiping [2], and channel filtering [1]. 


\section{Acknowledgments}

This research has been funded in part by the AFOSR grant FA9550-07-1-0039 and the AFOSR MURI grant FA9550-08-1-0356. This material is based upon work supported under a National Science Foundation Graduate Research Fellowship.

\section{References}

1. F. Bourgault and H Durrant-Whyte. Communication in general decentralized filter and the coordinated search strategy. In Proc. of FUSION'04, 2004.

2. Stephen Boyd, Arpita Ghosh, Balaji Prabhakar, and Devavrat Shah. Randomized gossip algorithms. IEEE/ACM Trans. Netw., 14(SI):2508-2530, 2006.

3. L. Chaimowicz and V. Kumar. Aerial shepherds: Coordination among uavs and swarms of robots. In 7th International Symposium on Distributed Autonomous Robotic Systems, 2004.

4. J. L. Drury, J. Richer, N. Rackliffe, and M. A. Goodrich. Comparing situation awareness for two unmanned aerial vehicle human interface approaches. In Proceedings of the IEEE International Workshop on Safety, Security and Rescue Robotics, 2006.

5. C. V. Goldman and S. Zilberstein. Optimizing information exchange in cooperative multiagent systems. In Proceedings of the Second International Conference on Autonomous Agents and Multi-agent Systems, 2003.

6. Wendi Rabiner Heinzelman, Joanna Kulik, and Hari Balakrishnan. Adaptive protocols for information dissemination in wireless sensor networks. In Proc. of MobiCom'99, 1999.

7. D. Kuokka and L. Harada. Matchmaking for information agents. Readings in Agents, 1995.

8. Daniel Kuokka and Larry Harada. Readings in Agents, chapter Matchmaking for information agents. Morgan Kaufmann, 1997.

9. CC Leary, M. Schwehm, M. Eichner, and HP Duerr. Tuning degree distributions: Departing from scale-free networks. Physica A: Statistical Mechanics and its Applications, 382(2):731738, 2007.

10. Ranjit Nair and Milind Tambe. Communication for improving policy computation in distributed pomdps. In Proc. of AAMAS'04, 2004.

11. M. Rosencrantz, G. Gordon, and S. Thrun. Decentralized sensor fusion with distributed particle filters. In Proceedings of the Conference on Uncertainty in AI (UAI), 2003.

12. Maayan Roth. Execution-time Communication Decisions for Coordination of Multi-agent Teams. PhD thesis, Robotics Institute, Carnegie Mellon Univ., Pittsburgh, PA, 2007.

13. N. Schurr, J. Marecki, M. Tambe, P. Scerri, J.P. Levis, and N. Kasinadhuni. The future of disaster response: Humans working with multiagent teams using DEFACTO. In AAAI Spring Symposium on Homeland Security, 2005.

14. Milind Tambe. Agent architectures for flexible, practical teamwork. National Conference on AI (AAAI97), pages 22-28, 1997.

15. P. Velagapudi, O. Prokopyev, K. Sycara, and P. Scerri. Maintaining shared belief in a large multiagent team. In In Proceedings of FUSION'07, 2007.

16. Prasanna Velagapudi, Oleg Prokopyev, Katia Sycara, and Paul Scerri. An analysis of information sharing in large teams. In Proceedings of AAMAS'09, 2009.

17. Y. Xu, P. Scerri, B. Yu, S. Okamoto, M. Lewis, and K. Sycara. An integrated token-based algorithm for scalable coordination. In AAMAS'05, 2005.

18. P. Xuan, V. Lesser, and S. Zilberstein. Communication decisions in multi-agent cooperation: Model and experiments. In Proceedings of the Fifth International Conference on Autonomous Agents, 2001. 